\title{
Dyslexia and a temporal processing deficit: A reply to the commentaries
}

\author{
RAYMOND M. KLEIN \\ Dalhousie University, Halifax, Nova Scotia \\ and \\ MARY E. FARMER \\ IWK Children's Hospital, Halifax, Nova Scotia, Canada
}

\begin{abstract}
A number of points and criticisms were raised in the commentaries on our review paper (Farmer \& Klein, 1995), and in this reply we address the most pertinent and major of those points. First, we clarify and expand upon what we mean by a temporal processing deficit. We then address StuddertKennedy and Mody's (1995) major claims, which are confined to the auditory modality, that (1) a discriminative deficit underlies what they see as a rate of processing deficit, and (2) discriminative/rate deficits for speech and nonspeech materials are independent. We explain why we believe the first proposal is unlikely to provide an explanation of the temporal processing deficits that we reviewed, and we present a simple framework within which speech and nonspeech perceptual codes are viewed as higher level isolable subsystems that depend on a common, lower level, auditory input system. The speech and nonspeech systems may be influenced similarly by damage to, or impairments of, their common input system, but they can be selectively influenced by insults after the pathways diverge. Then we address some of the issues raised by Rayner, Pollatsek, and Bilsky (1995), relating to visual deficits and oculomotor behavior, and we point to the rapidly growing evidence to diminish skepticism about the occurrence of a transient system deficit in dyslexia. Next, while agreeing that case studies are valuable, we dispute Martin's (1995) endorsement of the case study as the preferred methodology for studying a heterogeneous deficit such as developmental dyslexia. Finally, we affirm our original conclusion that more research aimed at revealing the nature and generality of the visual and auditory temporal processing deficits is warranted, and we reiterate some of our suggestions for the types of study that might help elucidate if and how these deficits might be causally related to the dyslexia with which they are frequently associated.
\end{abstract}

Martin (1995), Rayner, Pollatsek, and Bilsky (1995), and Studdert-Kennedy and Mody (1995) have written some interesting commentaries in response to our review paper outlining the evidence for a temporal processing deficit in some cases of dyslexia. Studdert-Kennedy and Mody's major emphasis is their criticism of the work of Paula Tallal (whose studies, incidentally, have been carried out mostly with language-impaired children), and not of our paper, with its focus on dyslexia and the confluence of evidence from a variety of researchers and paradigms. In our view, the Studdert-Kennedy and Mody commentary demonstrates a misunderstanding of both Tallal's work and our interpretation and expansion of Tallal's hypothesis. In responding to their criticisms, we will focus on our position and try to correct any inconsistencies that may have contributed to this misunderstanding. Given the nature of their commentary, a separate reply by Tallal is clearly warranted. Although the other two commentaries (Martin, 1995, and Rayner et al., 1995) digress

The authors would like to thank Dennis Phillips for his feedback on the ideas in this commentary. Correspondence should be addressed to R. M. Klein, Department of Psychology, Dalhousie University, Halifax, NS, Canada B3H 4J1 (e-mail: klein@ac.dal.ca or farmer@ac.dal.ca). somewhat from our main themes in directions of particular interest to the authors, the issues that they raise are relevant to some of our claims. In this reply to the commentaries, we will first address the question of what we mean by a temporal processing deficit and reemphasize our contention that such a deficit in the auditory modality would affect the perception of both speech and nonspeech stimuli. We will then discuss the major points raised by individual commentators.

As we emphasized in our paper, dyslexia is a symptom that clearly results from a myriad of different causes, and no one explanation or etiology will be found to explain the large number of cases that exist. ${ }^{1}$ In this respect, it is important to keep in mind that the ultimate aim of any research into reading difficulties must continue to be not the single-minded promotion of one hypothesis to explain dyslexia, but the furthering of our understanding of what might be causing the reading disability in individual dyslexic children and an attempt to find the most appropriate ways to help each child to learn to cope with or overcome the reading disability. We hope that our paper, by virtue of stimulating further research, will prove to be another step on the road to a more complete understanding of reading disabilities. 
We set out to accomplish several aims in our review paper. One was to break down temporal or sequential processing into logically distinct components operationalized in the performance of particular tasks. The components that we identified were individuation, temporal order judgment (TOJ), and discrimination of sequences. We prefaced our operational definition of the proposed components with a discussion of detection/ identification of individual stimuli, not because we view this as explicitly temporal/sequential, but because we saw this as a prerequisite for the components that we identified. We reviewed studies in which these components were assessed and found that in many studies performance of the dyslexic group was impaired in relation to that of the normal reading group. We thus felt that we had demonstrated that there were sufficient grounds to investigate further both the nature and the prevalence of temporal processing deficits. Finally, we speculated how a temporal processing deficit, in either or both of the auditory and visual modalities, might be causally related to reading disabilities.

\section{What Do We Mean by Temporal Processing Deficit?}

Since Rayner et al. (1995) found our notion of a temporal processing deficit vague, and Studdert-Kennedy and Mody appear to think that we should have meant something else given the terminology that we chose, let us begin by clearing up this confusion. When we first introduced Tallal's hypothesis, we referred to a "general deficit in processing rapid temporal sequences" (p. 462), and in the next section (pp. 462-463), following and expanding upon Hirsh and Sherrick (1961), we broke temporal/sequential processing down into a "logical sequence of the progressively more complex processes that might be said to fall under this rubric." We noted that detection and identification are a prerequisite for some of the tasks (individuation, ordering) that we would review, but that they do not necessarily involve processing a sequence. Thus, for the purposes of our review, we had a very clear (not vague) operational definition of temporal processing in terms of the ability to individuate or separate two closely spaced (in time) identical stimuli and the ability to perceive the order of two or more items presented in temporal proximity. We never used the phrase "temporal perception," which does connote the perception of temporal properties, and it is disingenuous when Studdert-Kennedy and Mody equate temporal perception with temporal processing and then decide that we must have meant what they think the former should mean, particularly when we so clearly defined (operationally speaking) what we did mean.

Our review shows that dyslexics, as a group (a phrase we will not continue to use, but always mean), are impaired in relation to normal controls on individuation tasks (i.e., they require more time separating two stimuli to detect the gap or to report that two stimuli have been presented) and on temporal order tasks, as long as the items are brief and closely spaced in time. Operationally speaking, that is the deficit. Dyslexics appear to be unimpaired in the detection of single stimuli and in the identification of such stimuli - unless identification is dependent on complex rapid changes over time (as would be the case with the consonant-vowel [CV] syllable identification studies Studdert-Kennedy and Mody, p. 511, incorrectly think we should have included in Table 1).

Demonstrating that dyslexics have a temporal processing deficit as defined operationally by performance on particular tasks does not, we agree, say much about the underlying nature of the deficit. We were deliberately vague about the underlying nature of the temporal processing deficit, primarily because "its full nature, origin and extent remain to be determined" (which is precisely how Studdert-Kennedy and Mody describe the nature of the widely accepted phonemic deficit, p. 513), and our main conclusion is that more resources should be devoted to discovering its nature. One interpretation of the nature of the performance deficit revealed in our review is in terms of rate of processing. For example, somewhere along the pathway from sensory receptors to perception there may be a stage or system that cannot accurately encode or track a signal that is changing very rapidly or is followed closely by another stimulus in time. This appears to be Tallal's position. (As an aside, Studdert-Kennedy and Mody in their abstract agree that a rate of processing deficit may characterize many dyslexics. However, they disagee with our interpretation of Tallal's position in two respects: ${ }^{2}$ They believe that discriminative capacity, rather than rate of processing, is primary, and that the deficit with nonlinguistic stimuli is independent of the deficit with linguistic stimuli. We will address both points in the next section.) Other interpretations are possible. One might be that encoded information is "smudged" over time, or in other words, that the mental representation loses some of the temporal crispness of the actual input. This interpetation seems most consistent with the individuation data, and in vision this seems to be one interpretation that has been attributed to a transient system deficit (see, e.g., Figure 8 from Breitmeyer, 1980). A third interpretation is that the times assigned to events and/or their properties are more variable (which might be referred to as temporal jitter). Temporal jitter could be present at every stage or level of processing and could be responsible for smudging if onsets and offsets jitter independently. A related idea would be to extend Treisman's view (Treisman \& Gelade, 1980) about the role of attention in the conjoining of attributes to their spatial locations to the conjoining of attributes to their time of occurrence. This extension would imply that some of the performance deficits that we described might be attributable to a specific form of attentional difficulty - an inability to focus attention in time, which would result in the jittery assignment of encoded identities along the time dimension. These interpretations, which are not mutually exclusive, are purely speculative attempts to explicate, in information-processing terms, 
the nature of the deficit revealed in our review. They can be regarded as food for thought and a stimulus for experimentation.

\section{Is the Temporal Processing Deficit in Audition Temporal and General?}

Studdert-Kennedy and Mody argue that the observed deficits in dyslexics in the rapid identification of phonetic or tone stimuli "stem from independent deficits in speech and nonspeech discriminative capacity, not from a general deficit in rate of auditory perception" (abstract). The two separate claims contained here are addressed in their paper. One is that the observed deficits in rate of processing are due to an underlying deficit in discriminative capacity; the second is that the deficits in speech and nonspeech are independent.

We proposed that a temporal processing deficit in audition may cause a deficit in the encoding of speech signals and, consequently, "less sharply defined" phonological categories. By suggesting that a discrimination deficit may be primary, Studdert-Kennedy and Mody have reversed the direction of causality. The finding of less consistent identification of CV stimuli by impaired readers (see Studdert-Kennedy \& Mody, p. 511) is cited in this context, but, by itself, this finding is equally consistent with both positions. However, because rapid acoustic changes are involved along the entire $\mathrm{CV}$ continuum, the additional finding that in some of these studies " $\mathrm{im}$ paired readers performed significantly worse than normal controls between categories but not within" (p. 511) appears to argue against the temporal processing deficit explanation. One counterargument might begin with a speculative extension of the developmental finding that exposure to one's native language and acquisition of its phonology results in a loss of discriminability across boundaries that are not phonemic in one's native language (Kuhl, 1992; Werker, 1989). We hypothesize that exposure to one's native language and acquisition of its phonology result in a reduced ability in the normal listener to discriminate within a phonemic category. Were this the case, the discrimination performance (within a phonemic category) of individuals suffering from a rate of auditory processing deficit would reflect counteracting effects in relation to that of normal individuals: a decrement (as with the between-category stimuli) due to the temporal processing deficit and an increment directly related to their poorer acquisition of the category. When the discrimination performance of reading-impaired individuals is examined without reference to the normal group, sensitivity along the $/ \mathrm{ba} /-/ \mathrm{da} /$ and $/ \mathrm{ba} /-/ \mathrm{ga} /$ continua has been shown to be relatively continuous, with no special deficit in the boundary region (see Godfrey, Syrdal-Lasky, Millay, \& Knox, 1981, Figure 4). Hence, this pattern (of dyslexics being worse than normals between but not within a phonemic category) does not provide definitive evidence against a temporal processing deficit explanation of the CV identification data. Finally, when the phonemic contrast is signaled by voice onset time, there is evidence (Steffens, Eilers, Gross-Glenn, \& Jallad, 1992) not only that dyslexic readers are more variable in their identifications, but that they require a longer period of silence in order to achieve the voiceless percept than do normal readers. A discriminative capacity deficit in the temporal dimension would seem to be necessary in order to explain this finding.

One retort to the suggestion that the underlying deficit might be in discriminative capacity is that the performance deficit in TOJs depends on the temporal proximity of the stimuli, a finding that is predicted by a rate of processing deficit but not by a discrimination deficit. Studdert-Kennedy and Mody's reply, that "the TOJ task is primarily a diagnostic tool for picking up subtle deficits in discriminative capacity, and these deficits reveal themselves in a slowed rate of perception, specific to the dimension being tested" (p. 509) merely redescribes the assertion that the discrimination deficit is primary without providing any explanation.

Hoping that it does not damage our position too much (but being more interested in knowing the truth than in being correct), we suggest the following explicit causal connection between a hypothetically primary discrimination deficit and a consequent rate of processing deficit. When assigning identities to stimuli from a particular set, the number of samples of evidence required in order to achieve a particular level of accuracy varies directly with the similarity of the alternatives. Hence, as the interval between an item being identified and a subsequent item is decreased, thus reducing the time (sampling opportunities) for the identification of the first item, performance on that item should deteriorate as the similarity between the stimuli in the set increases. Individuals who have what Studdert-Kennedy and Mody refer to as less discriminative capacity on the dimensions necessary for identifying those stimuli (as many dyslexics appear to have with phonology) will experience greater perceptual similarity between pairs of stimuli from that dimension than would be experienced by individuals with better discriminative capacity.

There are several reasons why we doubt such an explanation of the temporal processing deficits that we reviewed. First, and foremost, a discrimination-based temporal processing deficit cannot account for the deficit in individuation tasks (where subjects are asked whether there is a gap, or whether there are one or two stimuli) shown in Table 2 from Farmer and Klein (1995). We assert that the only dimension whose reduced discriminative capacity could account for these findings is time!

Second, the bulk of the temporal order errors in our own data (Farmer, 1993) cannot be explained away in terms of identification errors. Our task was modeled on that used by Tallal (1980), who called it repetition, and the same task was also used by Reed (1989). In this task, subjects are first trained to attach two buttonpress responses to two different tones (we will call them high, $\mathrm{H}$, and low, L). Then they are presented with a series of trials consisting of two tones separated by a relatively 
Table 1

Proportion of Responses of Each Type to Each of the Four Possible Stimulus Sequences

\begin{tabular}{ccccc}
\hline & \multicolumn{4}{c}{ Response } \\
\cline { 2 - 5 } Stimulus & HH & LL & HL & LH \\
\hline HH & 0.921 & 0.079 & 0.000 & 0.000 \\
LL & 0.128 & 0.872 & 0.000 & 0.000 \\
HL & 0.000 & 0.075 & 0.700 & 0.225 \\
LH & 0.000 & 0.075 & 0.325 & 0.600 \\
\hline
\end{tabular}

Note-The stimuli were $100-\mathrm{Hz}(\mathrm{L})$ and $305-\mathrm{Hz}(\mathrm{H})$ tones, each $75 \mathrm{msec}$ in duration separated by a $40-\mathrm{msec}$ ISI. The subjects were 20 dyslexics with a mean age of 14 whose reading averaged five grade levels below normal. It should be noted that responses along the positive diagonal are correct and all other responses are erroneous. (Derived from Farmer, 1993.)

short interstimulus interval (ISI) and are instructed to respond with two successive buttonpresses corresponding to the relative pitch of the first and second tones. The row and column labels in Table 1 show what the possible stimulus sequences and responses could be, and the 16 cell matrix represents the possible stimulus/response combinations in this task. This simple matrix is very instructive, because there are several different types of perceptual error, only two of which can be construed as order errors, and eight of which would yield an error if the task were same/different discrimination. Yet, as far as we know, all previous investigators who have used this task report a single, "total" error rate. There are several points about the data shown here which challenge the view that order errors are due to identification errors. First, although there are misidentifications (about $10 \%$ when the two stimuli are the same, with two high tones reported as two low ones and vice versa), order errors due only to misidentifications would require two misidentifications on the same trial and the probability of this occurring is about $1 \%$. The actual rate of order errors is about $27 \%$ in this data set (which is drawn from the shortest ISI [40 $\mathrm{msec}$ ] used by Farmer, 1993). Second, note that the $7.5 \%$ misidentifications that occur on trials with different identities (HL, LH) are not primarily misidentifications of the first stimulus, as would be predicted if the short interval before the occurrence of the second stimulus did not permit sufficient time for identification of the first one. Third, note that, overall, the rate of perceptions that would correspond to an error if the task had been to determine whether the two stimuli were the same or different is very low (averaging $3.75 \%$ across all four stimuli). The latter pattern causes us to be concerned about the reliability of the high same/different error rates reported by Tallal (1980) in her study of dyslexics. In this regard, we know of no other study similar to Tallal's in which dyslexics have been tested with these tone sequences in a same/different task.

The most direct way to assess the discriminability of items along a dimension or in a domain, when there is enough time to avoid the direct contribution of a rate of processing deficit, is to ask subjects to identify physi- cally similar items presented singly. Of course, one would want to avoid items (such as CV syllables) for which, because of the presence of a rapidly changing acoustic signal, ${ }^{3}$ the alternative temporal processing hypothesis makes the same prediction. Our review of studies of the detection or identification of single stimuli (Farmer \& Klein, 1995, Table 1) did not reveal a deficit, but it could be that none of the studies reviewed used stimuli that were sufficiently similar.

Before reviewing the evidence against StuddertKennedy and Mody's other claim, that the deficits in speech and nonspeech are independent, let us make a few points abundantly clear. We do not dispute the fact that areas of the dominant (usually left) hemisphere are specialized for speech and language. We believe that some of this specialization involves the categorization of phonemes. However, we also believe that prior to the categorization of phonemes, the sound signal composing a spoken message must be accurately encoded. This encoding takes place in pathways that are not specialized for speech, but that are necessary for the encoding of all auditory stimuli. Certain patterns of auditory input are then extracted and categorized phonemically in a "speech area." Other patterns, however, may not activate perceptual codes in this speech area, but instead may activate perceptual codes in other nonspeech areas to which the auditory system likewise projects.

Our view can be illustrated best with reference to the simple diagram in Figure 1. At the earliest stages of auditory analysis, which precede, and provide the raw material for, what we would call perception, there is no distinction between speech and nonspeech signals. At some point, processing diverges, and isolable speech and nonspeech perceptual codes are produced. The reader may notice a similarity between this view and Posner's (1978) notion that the physical and name codes of a visually presented letter are isolable (not independent) subsystems,

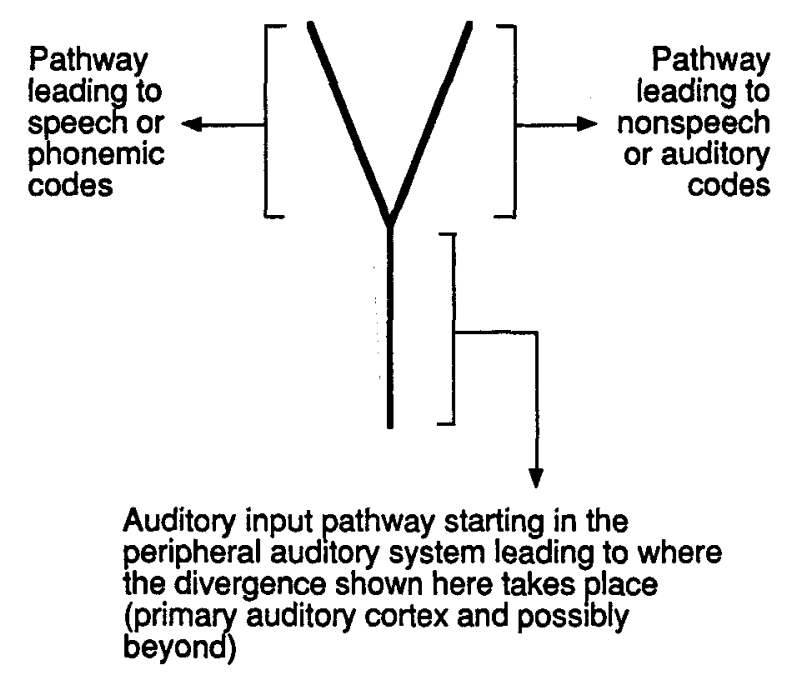

Figure 1. Simplified diagram showing flow of information in the auditory system. 
both arising from earlier, shared visual processing stages. On this view, damage to the early auditory stages will obviously result in deficits for both speech and nonspeech material and might be misinterpreted as evidence for a complete dependence between the speech and nonspeech systems or against a special speech system. Tallal's hypothesis involves a low-level deficit early in the common auditory input pathway that is characterized by an inability of the system to keep up with, or track, rapid changes in the acoustic signal. We also hypothesize a deficit in the common input pathway, but we would like to see more research before choosing among the interpretations (the rate of processing, temporal smudging and temporal jitter) that we described earlier (pp. 460461). Conversely, localized impairment after the divergence, to either the speech system or the nonspeech system, would produce a selective deficit that might be misinterpreted as evidence for complete independence of the two subsystems.

In supporting their hypothesis of the independence of deficits in speech and nonspeech auditory perception, Studdert-Kennedy and Mody rely heavily on their criticisms of Tallal and Newcombe (1978). Since we did not cite that paper in our review, we will not address the lengthy criticisms of it made by Studdert-Kennedy and Mody in their commentary on our paper. Suffice it to say that our arguments in favor of a general auditory temporal processing deficit, affecting speech and nonspeech alike in some dyslexics, were based, not on Tallal and Newcombe, but upon evidence from a number of investigations into auditory perception in both normal humans and primates, and from studies investigating the correlation of performance on temporal processing tasks using nonspeech stimuli with performance on both speech perception and reading tasks.

Much of this evidence has already been outlined in our review paper (e.g., pp. 480-483), and we do not intend to reiterate it here in detail. In our first line of argument, we reviewed evidence for categorical perception of nonspeech and speech sounds in infants and in primates, and noted that a number of researchers (e.g., Jusczyk, Rosner, Reed, \& Kennedy, 1989; Kuhl, 1992) have concluded that it is the basic (and general) physiological properties of the auditory processing system that has allowed language to develop, rather than any "prewired" speech processing mechanisms. Further evidence in this regard comes from a recent study by Steinschneider, Schroeder, Arezzo, and Vaughan (1995), who examined auditory evoked potentials in monkeys exposed to $\mathrm{CV}$ syllables differing only in voice onset time (VOT). Their results suggested that $\mathrm{Al}$ responses in the monkey can be differentiated for $/ \mathrm{ta} /$ and $/ \mathrm{da} /$ in that transient responses for the relatively short $(0-20 \mathrm{msec})$ VOT for $/ \mathrm{da} /$ were suppressed, while transient responses to /ta/ (with VOTs of $40-60 \mathrm{msec}$ ) were not, because they occurred after the hyperpolarization period. Steinschneider et al. noted that the change from suppression to nonsuppression occurred at a VOT of $20-40 \mathrm{msec}$ and thereby provided a physio- logical explanation for an acoustically based categorical boundary that is observed for speech sounds such as voiced and unvoiced stop consonants. On the basis of their results, Steinschneider et al. suggest that voiced and unvoiced consonants which straddle this boundary should be easily discriminated, whereas differences in VOT on either side of the boundary should not. They also concluded that such an acoustically based boundary "supports the more fundamental premise that the distinction between voiced and unvoiced stop consonants is partially based upon the ability to sequence the temporal order of acoustic events" (p. 337). The Steinschneider et al. study did not involve nonspeech stimuli, but a study by Molfese and Molfese (1985) did. They found that children who demonstrated different levels of linguistic skills at the age of 3 years had been differentiated when newborn by their auditory evoked responses to both CV speech syllables and to sine waves matched to the formant frequencies of the speech sounds. They suggested that the ability to make finer discriminations between auditory events, both speech and nonspeech, enabled their linguistically skilled group to utilize the information gleaned from such discriminations in developing language acquisition. While this does not demonstrate that the shared deficit was temporal, it is certainly consistent with such a view and moreover argues for an association between speech and nonspeech auditory functioning early in development. Finally, we would direct the reader to review our description of the Stefanatos, Green, and Ratcliff (1989) study (p. 483 of our review paper), in which receptive dysphasic children showed virtually flat evoked responses to frequencymodulated tones, in contrast to the responses of expressive dysphasics and normal controls. Stefanatos et al. concluded that their results indicated that a general difficulty with processing rapid changes in frequency at a basic sensory level contributed to the receptive dysphasics' speech perception impairments. This result, from the developmentally language-impaired literature, simply underscores our point that the early perceptual processing of rapid auditory changes is an essential first step before categorization of phonemes, and then perception of speech, can take place.

A second line of argument in our review paper involved correlations of performance on temporal processing tasks involving both speech and nonspeech stimuli, with each other and with performance on reading-related tasks. In some studies, correlations between speech and nonspeech tasks were not reported but may have been present, because reading-disabled groups are impaired on both types of task (e.g., Reed, 1989). Studies such as those reported in Tallal (1980), Farmer and Klein (1993), and Kinsbourne, Rufo, Gamzu, Palmer, and Berliner (1991) did not include temporal processing tasks with speech stimuli, but these authors did report significant correlations between a variety of temporal processing tasks with nonspeech stimuli and a number of reading and readingrelated tasks. In a study that Studdert-Kennedy and Mody 
have brought to our attention ${ }^{4}$ (Watson \& Miller, 1993), highly significant correlations between a nonspeech (tone) temporal order task and tasks measuring reading, phoneme segmentation, and speech perception were obtained in a sample of 24 reading-disabled and 70 nondisabled college students. Tallal (1980) reported that dyslexics' performance on her TOJ task was highly correlated (.81) with performance on a task of reading nonsense words. If the severity of the reading disorder in Tallal's dyslexic children is due, immediately, to their poor phonological perception/representation, then a correlation of this magnitude between nonverbal TOJ performance and reading nonsense words is certainly hard to reconcile with Studdert-Kennedy and Mody's view that deficits in rate of processing nonspeech and speech stimuli are independent. Studdert-Kennedy and Mody have suggested that such results may be apparent because the poor readers in such studies have an auditory temporal processing deficit for nonspeech stimuli that is independent of linguistic processing. However, since they believe that most if not all dyslexics have a speech (phonological) deficit, the assertion that the speech and nonspeech deficits are independent seems implausible.

Studdert-Kennedy and Mody accuse us of hoping "to evade the results of Aram and Ekelman (1988)" (p. 513), an experiment which they regard as a critical test and which they present to support their "independence" viewpoint. As we noted clearly in our footnote referring to that study, the subjects studied were not developmentally language impaired and there was no evidence of any of them having premorbid difficulties with language. Aram and Ekelman clearly stated that the difficulties following their subjects' unilateral brain lesions were in higher level language and learning; no mention was made of phonological or speech perception difficulties. Thus, there is no reason whatsoever to expect that these left-hemisphere-lesioned children would have difficulty with temporal processing tasks. That areas of the left hemisphere are dedicated to speech and language is not in dispute. If there is an area of the left hemisphere that is dedicated to perception of rapidly incoming auditory stimuli (be it primary auditory cortex or some other area) and that projects to the speech area, it will of course be relatively small in the great scheme of the left hemisphere. It is unwarranted to assume that any unspecified "left hemisphere" damage will of necessity affect the processes performed by that small area. Thus, the suggestion that Aram and Ekelman's (1988) results "disprove" our (or Tallal's) hypothesis is clearly untenable.

Given the electrophysiological evidence outlined above in support of a general auditory processing system whose efficacy affects the processing of all sounds with rapid changes, the argument for a temporal processing deficit that incorporates speech and nonspeech sounds appears to be more plausible. Clearly, methodologically rigorous studies with tasks in which both speech and acoustically equated nonspeech stimuli are presented to reading-disabled children are needed for this hypothesis to be investigated.
Studdert-Kennedy and Mody claim that such a study now exists. They describe an unpublished study by Mody, Studdert-Kennedy, and Brady (1995; based on Mody's, 1993, dissertation), in which "reading-impaired" subjects and controls were tested for discrimination and TOJ of $/ \mathrm{ba} /-/ \mathrm{da} /$ pairs and $/ \mathrm{ba} /-/ \mathrm{sa} /$ or $/ \mathrm{da} /-/ \mathrm{sa} /$ pairs at various ISIs, and also for discrimination of stimuli composed of two sine waves with durations and frequency trajectories matched to the $F 2$ and $F 3$ transitions in the $/ \mathrm{ba} /-/ \mathrm{da} / \mathrm{stimuli}$. They reported that their "readingimpaired" group was impaired on the /ba/-/da/ discrimination and TOJ tasks, as ISI decreased, but that performance on the nonspeech task was unaffected by decreases in ISI. In our view, this study, as a test of the auditory temporal processing deficit in dyslexia hypothesis, suffers from several problems. Most notable is the criterion for selection of the "reading-impaired" group, who were defined as "reading at least five months below grade level." Such a criterion does not meet any accepted definition of reading disability of which we are aware and thereby limits the ability to generalize the findings to dyslexia. A second point is that the subjects were given the TOJ task for the speech stimuli, but not for the nonspeech stimuli. Why was this obviously equated task omitted? Although the nonspeech stimuli used by Mody et al. were cleverly constructed, they may not be the "appropriate nonspeech control" stimuli that StuddertKennedy and Mody point out as being so necessary. It is possible, for example, that the steady-state pure sine waves (which follow the frequency trajectory) are less efficient at masking the rapid changes of the preceding trajectory than the formants in real or synthesized speech, by virtue of the fact that the latter contain acoustic energy over a relatively wide range of frequencies. In order to provide a better controlled comparison, we suggest the use of CVC syllables presented normally (speech) and backward (nonspeech). Another suggestion would be to construct $\mathrm{CV}$ stimuli with a consonant contrast, from a foreign language, that is not phonemic in the subjects' native language. Since both dyslexic and normal readers would be processing this material at a purely auditory level of analysis, Studdert-Kennedy and Mody's view that the dyslexics have a phonological discriminative capacity deficit predicts no group difference in either TOJ or discrimination, whereas the auditory temporal processing view predicts that the dyslexics would perform worse on both tasks. One final point is that the most obvious test of independence was not performed, or at least not reported: Was performance on the nonspeech task correlated with that on the equivalent speech task?

\section{Visual Transient System Deficit and Oculomotor Behavior}

The presence of two parallel channels or pathways in the visual system is now generally accepted (see, e.g., Livingstone \& Hubel, 1987). Anatomical, physiological, and psychological evidence supports a distinction that is often described functionally as transient versus sustained channels (Breitmeyer, 1980) and anatomically (in terms 
of the layers of the lateral geniculate nucleus to which and from which the pathways project) as magnocellular versus parvocellular. It is worth noting that although there is a similar magno/parvo distinction between some cells in the auditory system, and there are cortical units with sustained and transient responses, there is as yet no evidence that the functional and anatomical differences in audition constitute pathways, or are even related. Our review, focused as it was on specific temporal processing tasks (individuation, temporal order, and sequence perception), did not present a comprehensive account of the co-occurrence of dyslexia with a transient system deficit in vision. ${ }^{5}$ We did, however, suggest that a transient system deficit might underlie some of the visual temporal processing deficits we observed (e.g., DiLollo, Hanson, \& McIntyre, 1983), and in developing a plausible causal link to dyslexia, we emphasized possible implications of the transient system deficit for reading.

Toward the end of their commentary (pp. 504-505), Rayner et al. (1995) point to several unrelated findings in the dyslexia literature that they claim have either failed to be replicated or have been demonstrated in only one laboratory. In this context, Klein, Berry, Briand, D'Entremont, and Farmer (1990) noted that the Geiger and Lettvin (1987) and Perry, Dember, Warm, and Sacks (1989) studies were methodologically flawed. When we (Klein et al., 1990) eliminated the flaws, we did not obtain their result (nor did Goolkasian \& King, 1990). Rayner et al.'s presentation of this as a nonreplication is a bit misleading, since we did not replicate their methods. Indeed, anyone who replicates their flawed methods will likely replicate their artifactual finding. We also feel that Rayner et al. (pp. 503 and 505) overemphasize the disputed eye movement findings of Pavlidis (1981, 1985), because we said nothing about how eye movements might be affected by the deficits that we reviewed; we certainly would not expect the "erratic" pattern reported by Pavlidis in his nonreading sequential fixation task; and the nonreplicability of this finding is certainly not relevant to our claims. An unfortunate side effect of the emphasis on Pavlidis, and on the other disputed or "unique" findings, is that it encourages the reader to think that the co-occurrence of a transient system deficit and dyslexia may not be replicable either.

Rayner et al.'s (1995) allusion to the work of Lovegrove (Lovegrove, Martin, \& Slaghuis, 1986), whose laboratory is arguably the most empirically productive advocate of the transient system deficit in dyslexia, in the context of all these "nonreplicated" ideas, is unfair and misleading. Numerous findings are consistent with a transient system deficit, and researchers from many laboratories have been involved in producing converging evidence (from psychophysical, psychophysiological, and anatomical methods) that such a deficit often cooccurs with dyslexia (DiLollo et al., 1983; Lehmkuhle, Garzia, Turner, Hash, \& Baro, 1993; Livingstone, Rosen, Drislane, \& Galaburda, 1991; and Williams \& LeCluyse, 1990 , are examples cited in our review; Cornellisen,
Richardson, Mason, Fowler, \& Stein, 1995, and Edwards, Hogben, Clark, \& Pratt, in press, are recent examples not cited there). The transient system deficit is certainly not a one-lab fluke.

Although oculomotor behavior is not a topic emphasized in our review, Rayner et al. (1995) make some interesting predictions for oculomotor behavior that might follow from two consequences of a visual transient system deficit that are mentioned in our review. Referring to the possibility that a transient system deficit might entail increased persistence from each fixation that would interfere with reading, Rayner et al. (1995) make the following prediction(s): "(1) dyslexics' eye fixations during reading may be longer than those for normal readers, or (2) dyslexics make more eye fixations than do normal readers" (p. 503). We agree that these are possible outcomes, but would add a third possibility: (3) oculomotor behavior may be relatively normal, but when it is, individuals with increased visual persistence due to a transient system deficit will consequently extract less information from each fixation. In our review, we imply the third possibility and also suggest that the negative consequences of a visual transient system deficit (e.g., hypothesized increased forward masking from the previous fixation) might make a task such as reading unpleasant for individuals with such a deficit, in which case they might avoid reading.

Consistent with their Prediction 1, Rayner et al. (1995) point out that many dyslexics do have longer fixation durations than normal readers have, but they also correctly note that other factors might increase fixation durations in dyslexics besides increased persistence (such as increased difficulty of the material). Thus, without converging evidence, this confirmatory observation is not particularly informative. Such evidence, for example, would be provided if dyslexics' fixation durations were longer than those of normal readers even when the material was equated for difficulty. In this context, Rayner et al. (1995) note that when Hyönä and Olson (1994, in press) equated difficulty of the reading task by using readingmatched, rather than age-matched, controls, there was no difference in fixation durations. Although this finding does converge upon the conclusion that increased fixation duration compared with that of age-matched controls might not be due to increased persistence, it is potentially compromised by the possibility that perceptual processes that might affect fixation duration (e.g., susceptibility to backward masking; Arnett and DiLollo, 1979) change with age. Perhaps an additional comparison, converging on the same conclusion, is still needed. This might involve dyslexic and normal readers who are the same age, reading words in text (requiring multiple fixations) which, when read singly, are read equally well by the two groups. However, even if difficulty in processing the words and not persistence from the prior fixation is responsible for dyslexic readers' longer fixation durations during reading, this merely represents an absence of positive evidence for the transient system defi- 
cit hypothesis, and not negative evidence. This is because, as noted above, individuals with increased persistence might have longer than normal fixation durations, but if they did not, they would extract less information in reading. The study by Shapiro, Ogden, and Lind-Blad (1990) discussed on p. 484 of our review paper, by showing that dyslexics have particular difficulty in reading words that might require multiple fixations, provides indirect support for the transient system deficit explanation of the increased fixation durations in dyslexics. More direct support for, or against, this explanation would have been available if oculomotor behavior had been recorded during Shapiro et al.'s experiment.

An alternative link between a transient system deficit and dyslexia mentioned in our review depends on the fact that the transient system predominates in peripheral vision, whereas the sustained system predominates in foveal vision. It has thus been hypothesized that a transient system deficit might entail a decreased or delayed contribution from information in the periphery during reading. The moving window technique developed by McConkie and Rayner (1975) provides an elegant way to test this idea, and the reading rates presented in Rayner et al.'s (1995) Figure 1 (adapted from Rayner, Murphy, Henderson, \& Pollatsek, 1989) as a function of window size and group would seem to support this hypothesis. Normals read faster than the dyslexics and benefit greatly from increases in window size up to three words; in contrast, dyslexics read much slower, benefit less from increasing window size, and most importantly do not benefit at all after the window size reaches two words. When this issue is examined by using fixation duration as a function of window size, the finding from the Rayner et al. (1989) study is similar (see their Figure 2). The fixation durations of normal readers are shorter, and they decrease as window size is increased. The dyslexics show a decrease in fixation duration as window size increases from one to two words but do not show a decrease as window size is increased further (suggesting that for the dyslexics information beyond two words in the periphery is not being utilized). The data from a similar study by Underwood and Zola (1986) do not show this pattern (see Rayner et al.'s, 1989, Figure 3). In particular, both groups show roughly the same decrease in fixation duration as window size is increased. This discrepancy can probably be explained in terms of methodological differences. Most importantly, Underwood and Zola's window is defined in terms of letters; Rayner et al.'s (1989) in terms of words. Rayner et al. (1995) conclude that the "usable" window extends to about 15 characters to the right, and Underwood and Zola's largest window size was 7 letters. It seems to us that if the dyslexics' function reached asymptote (representing the point in the periphery at which information was no longer useful in reading) somewhere between 7 letters and two words, then the results of both studies would be consistent with a transient system deficit. Another possibly important difference is that in Underwood and Zola's study, full text or the window alter- nated with successive saccades, a procedure not used by Rayner et al. (1995). Thus, on balance, the oculomotor findings reported by Rayner et al. (1995) are either neutral with respect to the transient system deficit (fixation durations are longer in dyslexics, but not with readingmatched subjects) or consistent with it (the usable window appears to be smaller in dyslexics).

Rayner et al. (1995) are justifiably concerned that the possiblity of a visual temporal processing deficit as a causal factor in dyslexia not become too exclusively the focus of attention, as they suggest that problems with reading caused by such a mechanism as we have postulated may be quite rare. As we pointed out in the review paper (p. 485) we are not in any way implying that a visual temporal processing difficulty (or a visual transient system deficit) is the sole or primary cause of reading problems in a large proportion of cases. We merely show how such a deficit could cause difficulty in reading; we suggest that such a deficit might add to the problems engendered by phonological difficulties; and we point out that because such a deficit might be symptomatic of a general temporal processing deficit (which has its effect on reading primarily through the auditory modality), it might be found to co-occur with dyslexia even when it does not have any direct causal link to the reading problem.

\section{Case Versus Group Studies and Relative Frequency of the Temporal Processing Deficit in Dyslexia}

Martin (1995) eloquently presents the argument for using single case, rather than group, studies when investigating developmental impairments. We agree with her that single case studies may provide invaluable information for building models of how the normal reading system develops and that they can contribute to what we know about the reading disability in the particular case being studied. Indeed, there are several benefits of the case study methodology. One is that an individual's performance profile is so well understood that an optimal remediation program can be designed for him/her. Another is that a particular case may present an existence proof for, or definitive counterexample to, a particular proposal. However, one danger with relying on case studies alone is that one may lose sight of the forest while looking closely at the trees.

Although we agree with Martin that case studies can be very valuable, we strongly disagree with her rejection of the group study methodology. We feel that group studies are especially informative in the initial stages of investigation. Although it is essential to know the characteristics of each individual poor reader in order to help that reader, it is equally essential that we know what sorts of questions we should be asking about each reader in the first place. As Martin (1995) points out in her commentary, having to test large numbers of children on many tasks, especially over a long period of time, is a daunting task for researchers. We would add that it is also daunting (and can be ethically questionable) to ask our young subjects (and their families) to make the sacri- 
fices in time and energy that would be required for such research, particularly if our probing was not empirically guided. It is the group studies that will hopefully give us an overview of what deficits are prevalent among what types of poor readers, and with what other deficits each may be correlated. Having thus mapped out the general plan of the forest, we can then begin to fill in essential details with single case studies for which the tasks have been chosen on the basis of known subject characteristics, rather than use a "shotgun" approach with tasks.

Martin implies that we are reluctant to endorse the case study approach. Our only reluctance is to endorse the case study approach to the exclusion of, or in preference to, group or other methods. Hegemony with regard to methodology should be avoided. Indeed, we believe that the most fruitful approach will involve the converging use of multiple methods, as we advocate in the penultimate section of our review paper and in Klein and McMullen (in press). As Martin has pointed out, the reason most of the studies cited in our review are group studies is that just such types of studies have directly explored the components of temporal/sequential processing in which we are interested. As we have pointed out (p. 476), single case studies that incorporate temporal processing tasks will now add to our knowledge of reading disability subtypes, and, although the possible developmental nature of temporal processing deficits will complicate any investigations, we also point out (e.g., p. 486) that groups of individual cases followed longitudinally are essential if we are to map the developmental course of such processing deficits.

Toward the end of her commentary Martin suggests two patterns that might be observed in case studies, either of which would undermine "the attribution of causation to the temporal processing disorder...: (1) a temporal processing disorder for nonspeech auditory stimuli, but no greater difficulty with stops than with vowels in perception or letter sounding, or (2) greater difficulty with stops than vowels, but no temporal processing disorder for nonspeech stimuli" (p. 498). No doubt these would be interesting cases to explore. Let us examine them in the context of the flow diagram in Figure 1. Note that either pattern could be explained in terms of impairments, resulting in a temporal processing deficit, located after the divergence into separate speech and nonspeech systems. Martin acknowledges this possibility for the second case, but not the first. If there were a reading disability in the first case, in which the nonspeech system was impaired while the speech system was intact, then we would look to a visual transient system deficit or a deficit in the connections from phonological to orthographic representations for the underlying cause. As such, the two cases Martin describes would not, by themselves, be decisive. Converging evidence showing that an abnormality was localized in the pathways prior to separation would be required (e.g., from ERPs that could reveal the chronometric locus of the deficit in the processing sequence or MRIs that could reveal the anatomical locus).
We concur with Martin (p. 486 of our review) that group studies such as that of Castles and Coltheart (1993) are invaluable for the analysis of individual differences in poor readers, while at the same time they can present information on subgroups of children who share certain characteristics. ${ }^{6}$ The good scientist will pay attention to subject differences (when these are not likely due to sampling error), and there are many valuable statistical/quantitative techniques for exploring them. Obviously when one is reporting group results, it is important to make clear what proportion of the group falls into the "normal" range and what proportion falls clearly outside this range. However, one must be careful when drawing conclusions from such a breakdown.

As an example, the Tallal (1980) report that 55\% (11 out of 20) of her disabled reader group scored within normal range in the temporal processing task in the presence of a significant group difference is cited by Martin to highlight the "danger" of group studies, and she claims that this finding makes it "unlikely that a temporal processing deficit was the source of the reading difficulties for the majority of the dyslexic subjects in that study" (p. 495; see also our caveat on p. 472 of the review paper).

A very important issue, one that is often neglected in this regard, is that the variability of a performance measure depends directly on the number of samples (trials per subject) that are obtained in an experiment. When the variability of the measure is high, there can be group overlap in the obtained scores even when there is absolutely none in the true scores. Since we, Tallal (1980), and Martin neglect this issue when discussing Tallal's data, that study serves as a particularly relevant and important example of this point. We conducted a simple simulation which revealed that the amount of overlap reported by Tallal cannot by itself be relied upon to support Martin's claim, primarily because, collapsed across all the short ISIs in her TOJ experiment, each subject provided only 24 trials. To illustrate our point, let us assume that: (1) two groups of subjects are tested on a particular task, (2) there is no between-subjects variability within either group (i.e., all dyslexics are homogeneous, as are all normals), and (3) the true proportions correct are .85 and .95 for the dyslexics and normals, respectively. Given these assumptions, it is obvious that none of the dyslexics would score in the normal range $(=.95)$ if enough samples of their performance (viz., trials) were collected. However, the variability of the proportion correct measure is such that with only 24 trials, approximately $5 \%$ of the normals will score below .85 , the mean of the dyslexic distribution, and thus slightly more than half of the dyslexics will score in the normal range (defined as better than the 5 th percentile). As this example makes abundantly clear, when one is making claims about whether an individual's performance is normal or not, it is essential that enough trials are collected per subject to make the variability of each subject's score small in relation to the mean difference between the groups. This was clearly not the case in Tallal's (1980) study. ${ }^{7}$ Of course the fact 
that it is often impractical to collect sufficient samples of performance on a single individual is one of the main reasons for group studies.

Thus, Tallal's oft quoted finding that $11 / 20$ (we must point out that in her discussion Tallal, 1980, refers to $12 / 20$, but the figure of $11 / 20$ is clear from her Results section) of her dyslexic subjects performed within normal limits cannot, by itself, be taken as evidence for heterogeneity within the dyslexic sample. Note that we are not by any means claiming that the dyslexics tested by Tallal or most other researchers were homogeneous (as we have pointed out on p. 476 and in numerous other places in our review paper), nor are we claiming that Tallal's dyslexics were not bimodally distributed in their TOJ performance. We merely make this point to illustrate that if an investigator wishes to make claims about the performance of individuals, it is essential to have enough data from each subject to keep the variance of the estimate sufficiently low. It is clear from reports of single case studies that results from many of the reading tasks can be extremely mixed, and that sometimes the performance of subjects on a number of tasks falls fairly close to the center of a continuum and thus does not allow for them to be classified unambiguously. Thus, just as one would use meta-analyses to grasp the larger picture when effect sizes are small in a number of studies, it is useful to look at group results with numbers of subjects whose individual performance may be difficult to classify clearly. Of course, it is necessary to do this when we wish to look at correlations between multiple measures or more generally to apply most multivariate techniques.

\section{Conclusion}

The three commentaries raise some interesting and provocative points about the temporal processing deficit hypothesis. However, after careful consideration of these points, we are not dissuaded from our original conclusion that the evidence available from a variety of paradigms is compelling enough to warrant further investigation into the temporal processing deficit and its possible causal role in a number of cases of dyslexia. Each commentary, in its own way, can be seen as responding to this call for "further investigation" (e.g., by seeking clarification of terminology, generating new predictions and testing them against existing data, suggesting and conducting "critical" experiments, and describing test cases) and, together with our review and reply, should stimulate research and thinking that will advance our understanding of the causes of developmental dyslexia.

Throughout our review paper, we raised a number of questions that need to be answered more fully for a proper evaluation of the temporal processing deficit hypothesis to be made. These include the need to investigate whether such a deficit is general (both across the auditory and visual modalities, and within the auditory modality, for both speech and nonspeech stimuli); and the need to explore whether a deficit in one component of temporal processing is necessarily linked to a deficit in other components. In addition, the questions of causality (auditory temporal processing deficit $\rightarrow$ phonemic deficit $\rightarrow$ dyslexia, and visual temporal processing deficit $\rightarrow$ dyslexia) need thorough investigation, as does (do) the possible developmental course(s) of a temporal processing deficit in the auditory and visual modalities. We suggested a number of directions that future research might take in search of the answers to the many questions raised. These include multivariate analytic methods; longitudinal studies; genetic and cross-cultural studies; anatomical and electrophysiological studies; and remediation studies. We emphasized the importance of designing methodologically sound studies with adequate controls, given the complexity of the deficit and the number of possible confounding variables. As is clear from the points and criticisms raised by the commentators, there are alternative explanations that may be invoked to explain results obtained, and it is imperative to design studies that can adequately distinguish among such alternatives.

\section{REFERENCES}

Aram, D. M., \& Ekelman, B. L. (1988). Auditory temporal perception of children with left or right brain lesions. Neuropsychologia, 26, 931-935.

ARNETT, J. L., \& DiLollo, V. (1979). Visual information processing in relation to age and to reading ability. Journal of Experimental Child Psychology, 27, 143-152.

BREITMEYER, B. G. (1980). Unmasking visual masking: A look the "why" behind the veil of the "how." Psychological Review, 87, 52-69.

Castles, A., \& Coltheart, M. (1993). Varieties of developmental dyslexia. Cognition, 47, 149-180.

Cornelissen, P., Richardson, A., Mason, A., Fowler, S., \& Stein, J. (1995). Contrast sensitivity and coherent motion detection measured at photopic luminance in dyslexics and controls. Vision Research, 35, 1483-1494.

DiLollo, V., Hanson, D., \& McIntyre, J. S. (1983). Initial stages of visual information processing in dyslexia. Journal of Experimental Psychology: Human Perception \& Performance, 9, 923-935.

Edwards, V. T., Hogben, J. H., Clark, C. D., \& Pratt, C. (in press). Effects of a red background on magnocellular functioning in average and specifically reading disabled readers. Vision Research.

FARMER, M. E. (1993). A comparison of the sequential processing abilities of dyslexic and normal readers, using visual and auditory tasks. Unpublished doctoral dissertation, Dalhousie University.

FARMER, M. E., \& KLEIN, R. (1993). Auditory and visual temporal processing in dyslexic and normal readers. In P. Tallal, A. M. Galabarda, R. R. Llinas, \& C. von Euler (Eds.), Temporal information processing in the nervous system: Special reference to dyslexia and dysphasia (Annals of the New York Academy of Sciences, Vol. 682, pp. 339. 341). New York: New York Academy of Sciences.

FARMer, M. E., \& KLEIN, R. M. (1995). The evidence for a temporal processing deficit linked to dyslexia: A review. Psychonomic Bulletin \& Review, 2, 460-493.

Geiger, G., \& LetTvin, J. Y. (1987). Peripheral vision in persons with dyslexia. New England Journal of Medicine, 316, 1238-1243.

Godfrey, J. J., Syrdal-Lasky, A. K., Millay, K. K., \& KNox, C. M. (1981). Performance of dyslexic children on speech perception tests. Journal of Experimental Psychology, 32, 401-424.

Goolkasian, P., \& KinG, J. (1990). Letter identification and lateral masking in dyslexic and average readers. American Journal of Psychology, 103, 519-538.

HiRsh, I. J., \& SHERrick, C. E., JR. (1961). Perceived order in different sense modalities. Journal of Experimental Psychology, 62, 423-432.

HYöNÄ, J., \& OLson, R. K. (1994). Dyslexic and normal readers' eye movement patterns in reading, visual search, and tracking. In J. Ygge 
\& G. Lennerstrand (Eds.), Eye movements in reading (pp. 233-244) Oxford: Pergamon Press.

HYöNÄ, J., \& OLSON, R. K. (in press). Eye fixation patterns among dyslexic and normal readers: Effects of word length and word frequency. Journal of Experimental Psychology: Learning, Memory, \& Cognition.

JusCZYK, P. W., RosNek, B. S., ReEd, M. A., \& KenNedY, L. J. (1989) Could temporal order differences underlie 2-month-olds' discrimination of English voicing contrasts? Journal of the Acoustical Society of America, 85, 1741-1749.

Kinsbourne, M., Rufo, D. T., Gamzu, E., Palmer, R. L., \& Berliner, A. K. (1991). Neuropsychological deficits in adults with dyslexia. Developmental Medicine \& Child Neurology, 33, 763-775.

Klein, R. M., Berry, G., Briand, K., D'Entremont, B., \& Farmer, M. [E.] (1990). Letter identification declines with increasing retinal eccentricity at the same rate for normal and dyslexic readers. Perception \& Psychophysics, 47, 601-606.

KLEIN, R. M., \& MCMULLEN, P. (in press). Converging methods for understanding reading and dyslexia. Cambridge, MA: MIT Press.

KuHL, P. K. (1992, September). The perception of speech by infants: What is given by nature, what is gained by experience. Paper presented at the New York Academy of Sciences/Rodin Remediation Academy Conference, New York

Lehmkuhle, S., Garzia, R. P., Turner, L., Hash, T., \& Baro, J. A. (1993). A defective visual pathway in children with reading disability. New England Journal of Medicine, 382, 989-996.

Livingstone, M. S., \& Hubel, D. H. (1987). Psychophysical evidence for separate channels for the perception of form, color, movement, and depth. Journal of Neuroscience, 7, 3416-3468.

livingstone, M. S., Rosen, G. D., Drislane, F. W., \& Galaburda, A. M. (1991). Physiological and anatomical evidence for a magnocellular defect in developmental dyslexia. Proceedings of the $\mathrm{Na}$ tional Academy of Sciences, 88, 7943-7947.

Lovegrove, W. J., Martin, F., \& Slaghuis, W. (1986). A theoretical and experimental case for a visual deficit in specific reading disability. Cognitive Neuropsychology, 3, 225-267.

MARTIN, R.C. (1995). Heterogeneity of deficits in developmental dyslexia and implications for methodology. Psychonomic Bulletin \& Review, 2, 494-500.

McConkie, G. W., \& Rayner, K. (1975). The span of the effective stimulus during a fixation in reading. Perception \& Psychophysics, $17,578-586$.

MoDY, M. (1993). Bases of reading impairment in speech perception: A deficit in rate of auditory processing or in phonological coding? Unpublished doctoral dissertation, City University of New York.

Mody, M., Studdert-Kennedy, M., \& Brady, S. (1995). Speech perception deficits in poor readers: Auditory processing of phonological coding? Manuscript submitted for publication.

MOLFESE, D. L., \& MOLFESE, V. J. (1985). Electrophysical indices of auditory discrimination in newborn infants: The bases for predicting later language development? Infant Behavior \& Development, 8, 197-211.

PAvLidis, G. T. (1981). Do eye movements hold the key to dyslexia? Neuropsychologia, 19, 57-64.

Pavlidis, G. T. (1985). Eye movement differences between dyslexic, normal, and retarded readers while sequentially fixating digits. American Journal of Optometry \& Physiological Optics, 62, 820832.

Perry, A. R., Dember, W. N., Warm, J. S., \& SaCks, J. G. (1989). Letter identification in normal and dyslexic readers: A verification. Bulletin of the Psychonomic Society, 27, 445-448.

Posner, M. I. (1978). Chronometric explorations of mind. Hillsdale, NJ: Erlbaum.

Rayner, K., Murphy, L. A., Henderson, J. M., \& Pollatsek, A. (1989). Selective attentional dyslexia. Cognitive Neuropsychology, 6. 357-378.

Rayner, K., Pollatsek, A., \& Bilsky, A. B. (1995). Can a temporal processing deficit account for dyslexia? Psychonomic Bulletin \& Review, 2, 501-507.

REED, M. A. (1989). Speech perception and the discrimination of brief auditory cues in reading disabled children. Journal of Experimental Child Psychology, 48, 270-292.
Shapiro, K. L., Ogden, N., \& Lind-Blad, F. (1990). Temporal processing in dyslexia. Journal of Learning Disabilities, 23, 99-107.

Stefanatos, G. A., Green, G. G. R., \& Ratcliff, G. G. (1989). Neurophysiological evidence of auditory channel anomalies in developmental dysphasia. Archives of Neurology, 46, 871-875.

Steffens, M. L., Eilers, R., Gross-GlenN, K., \& Jallad, B. (1992). Speech perception in aduit subjects with familial dyslexia. Journal of Speech \& Hearing Research, 35, 192-200.

Steinschneider, M., Schroeder, C. E., Arezzo, J. C., \& Vaughan, H. G. (1995). Physiologic correlates of the voice onset time boundary in primary auditory cortex (A1) of the awake morkey: Temporal response patterns. Brain \& Language, 48, 326-340.

STUART, A. (1994). A visual transient system deficit in developmental dyslexia: Psychophysical, visuospatial, electrophysiological, and anatomical evidence. Unpublished manuscript.

Studdert-Kennedy, M., \& Mody, M. (1995). Auditory temporal perception deficits in the reading impaired: A critical review of the evidence. Psychonomic Bulletin \& Review, 2, 508-514.

TAllal, P. (1980). Auditory temporal perception, phonics, and reading disabilities in children. Brain \& Language, 9, 182-198.

Tallal, P., \& Newcombe, F. (1978). Impairment of auditory perception and language comprehension in dysphasia. Brain \& Language, 5, 13-24.

Tallal, P., \& PierCy, M. (1973). Developmental aphasia: Impaired rate of non-verbal processing as a function of sensory modality. Neuropsychologia, 11, 389-398.

Tallal, P., Sainburg, R. L., \& Jernigan, T. (1991). The neuropathology of developmental dysphasia: Behavioral, morphological, and physiological evidence for a pervasive temporal processing disorder. Reading \& Writing, 3, 363-377.

Treisman, A., \& Gelade, G. (1980). A feature integration theory of attention. Cognitive Psychology, 12, 97-136.

UNDERWOOD, N. R., \& ZOLA, D. (1986). The span of letter recognition of good and poor readers. Reading Research Quarterly, 21, 6-19.

WATSON, B. U., \& Miller, T. K. (1993). Auditory perception, phonological processing, and reading ability/disability. Journal of Speech \& Hearing Research, 36, 850-863.

WERKER, J. (1989). Becoming a native listener. American Scientist, 77, 54-59.

Williams, M., \& LeCluyse, K. (1990). Perceptual consequences of a temporal processing deficit in reading disabled children. Journal of the American Optometric Association, 61, 111-121.

\section{NOTES}

1. In the very first sentence of their commentary, Studdert-Kennedy and Mody state that the "target paper starts from the widely accepted assumption (with which we agree) that dyslexia, or reading impairment, is often, if not always [our italics], associated with a phonological deficit" (p. 508). Studdert-Kennedy and Mody may believe that dyslexia may always be associated with a phonological deficit, but we do not. We have made it abundantly clear throughout our paper that dyslexia is a symptom of many possible etiologies, and that no unitary cause for reading impairment will be found.

2. Some quotations from Tallal, such as the one from p. 509 of Studdert-Kennedy and Mody's commentary: "difficulty with temporal pattern perception may stem from a more primary perceptual deficit that affects the rate at which they can process perceptual information" (Tallal, 1980, p. 193), may be subject to different interpretations. From this and similar quotes (Tallal \& Piercy, 1973, p. 396; Tallal, Sainburg, \& Jernigan, 1991, p. 365), Studdert-Kennedy and Mody infer that Tallal believes that a deficit in temporal sequencing tasks is caused by a rate-of-processing deficit, which in turn is caused by an underlying discrimination deficit that only becomes apparent under time pressure. In contrast, we interpret Tallal's (1980) clause "that affects the rate at which they can process perceptual information," and other similar statements, as defining the nature of the "primary perceptual deficit," rather than indicating one consequence of a discrimination deficit. We believe this interpretation to be more consistent with the corpus of Tallal's work. Nevertheless, because we and Studdert-Kennedy and Mody are so sure of our differing interpretations of what Tallal believes to be the underlying nature of the perceptual deficit, it would be timely for 
Tallal herself to resolve this issue. Finally, the implication of this discussion is that Studdert-Kennedy and Mody do not think that they disagree with Tallal about whether discriminative capacity is primary, and we think that they do.

3. In their section Perceiving Formant Transitions (pp. 511-512), Studdert-Kennedy and Mody present a number of arguments against our interpretation of a temporal processing deficit by quoting from p. 467 of our paper, in which we state that "any impairment in the ability to process the order of ... [spectral changes in stop consonants] would result in impaired discrimination of the sounds." We acknowledge that the wording of this sentence lends itself to a misinterpretation such as that made by Studdert-Kennedy and Mody. In keeping with the hypothesis propounded throughout our paper, we meant to convey that a difficulty in processing both the changes, and thus the order of changes, in any rapidly occurring auditory stimuli (such as the acoustic changes in stop consonants) would impair the ability to discriminate, and thus categorize, stimuli such as certain speech sounds. The end result would thus be a difficulty in discriminating between speech sounds such as stop consonant syllables, which are relatively similar. We defend our description of the formant transitions in speech, which are indeed changes in frequencies that take place very quickly over time, and thus are "a series of rapidly changing acoustic events," though we acknowledge that the term "events" could be easily misconstrued to imply awareness of them. Our argument is that such a processing difficulty would result in imperfect categorizations of certain sounds, and thus difficulty in discrimination of speech sounds such as stop consonants.

4. In describing the Watson and Miller (1993) study, StuddertKennedy and Mody correctly note that the impaired readers made significantly more errors than did normal readers on the tone TOJ task, whereas three other "temporal perception" tasks did not distinguish between the groups. It should be added that the normal readers performed better on each of these three tests than did the impaired readers (just not significantly so, using an alpha level of .004). The additional find- ing that TOJ performance was highly correlated with performance on the other three tasks that Watson and Miller used to assess auditory temporal processing (TBAC embedded tone $r=.56$, pulse train $r=$ .49 , and tonal duration $r=.44$, with $r=.29$ significant at .004 ) suggests that all four tasks were aimed at a similar underlying construct with the TOJ task being most sensitive. Although Studdert-Kennedy and Mody did not use Watson and Miller's LISREL analysis to support their arguments, it should also be noted that none of the structural equation models that Watson and Miller tested by using LISREL corresponds to the one proposed in our review, and by Tallal, which is that an auditory temporal processing deficit is responsible for a speech perception deficit that then impacts negatively on phonological awareness, reading, verbal short-term memory, and so forth. As such, we cannot be sure how such a model would have fared in fitting their data

5. Had we conducted such a review, we would have concluded that there was strong evidence for an association (see Stuart, 1994).

6 . The case/group distinction should probably be thought of as a continuum, with unambiguous examples (e.g., case-a single individual is explored intensively, usually in an effort to understand the mechanisms underlying his/her performance; group —one or more groups of individuals is [are] explored less thoroughly, usually in an effort to generalize findings to untested individuals who meet the group selection criteria) at the ends. In our view of this continuum, Castles and Coltheart (1993) is a group study that paid attention to individual differences. In Martin's view it is "a set of case studies carried out simultaneously."

7. It is impossible to assess this precisely on the basis of the information provided by Tallal (1980), but the numbers that we chose to illustrate this point agree with some of the information provided in her paper.

(Manuscript received July 28, 1995; revision accepted for publication August 7, 1995.) 